\title{
Thermal Conductivity and Stability of Hydrocarbon-Based Nanofluids with Palladium Nanoparticles Dispersed by Modified Hyperbranched Polyglycerol
}

\author{
Xiaomei Qin ${ }^{1 *}$, Shihao Yang ${ }^{1}$, Yapei Chen ${ }^{1,2}$, Xiaoyun Qin ${ }^{1}$, Jianbo Zhao ${ }^{1}$, Wenjun \\ Fang ${ }^{3 *}$, and Dan $\mathrm{Luo}^{2 *}$
}

${ }^{1}$ School of Material and Chemical Engineering, Zhengzhou University of Light Industry, Zhengzhou 450000, Henan Province, China

${ }^{2}$ State Key Laboratory of Heavy Oil Processing, College of New Energy and Materials, Beijing Key Laboratory of Biogas Upgrading Utilization, China University of Petroleum Beijing, Beijing 102249, China

${ }^{3}$ Department of Chemistry, Zhejiang University, Hangzhou 310027, Zhejiang Province, China

Preparation of dodecanethiol-coated Pd nanoparticles (Pd@S).

$\mathrm{K}_{2} \mathrm{PdCl}_{4}$ was dissolved in water, and $\mathrm{TOAB}$ was dissolved in toluene. The solution was mixed stirred for $1 \mathrm{~h}$. During the stirring process, the toluene phase gradually changed to be deep red. The toluene phase was collected, and 1-dodecanethiol was added in a mole ratio of $\mathrm{Pd}: \mathrm{C}_{12} \mathrm{H}_{25} \mathrm{SH}=1: 2$. $\mathrm{NaBH}_{4}$ was added dropwise into the organic phase. After the toluene phase became black, the solvent was removed. The black powder of Pd@S was obtained after washing with ethanol for three times. 


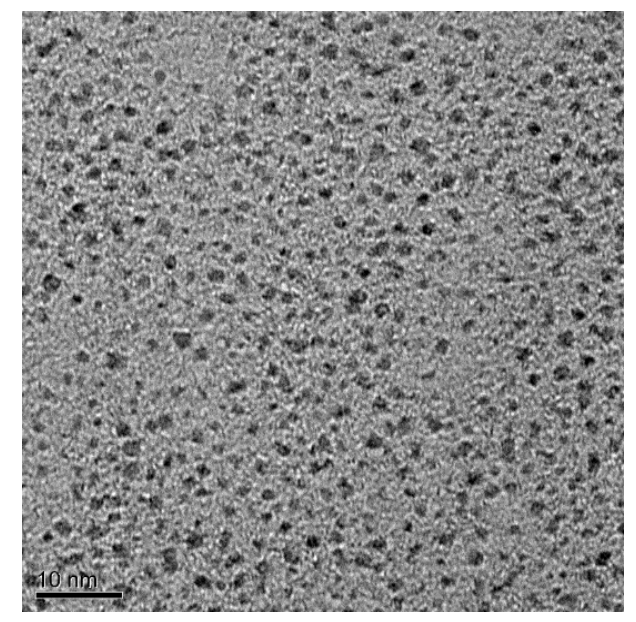

Figure S1. TEM image of $\operatorname{Pd@S.~}$
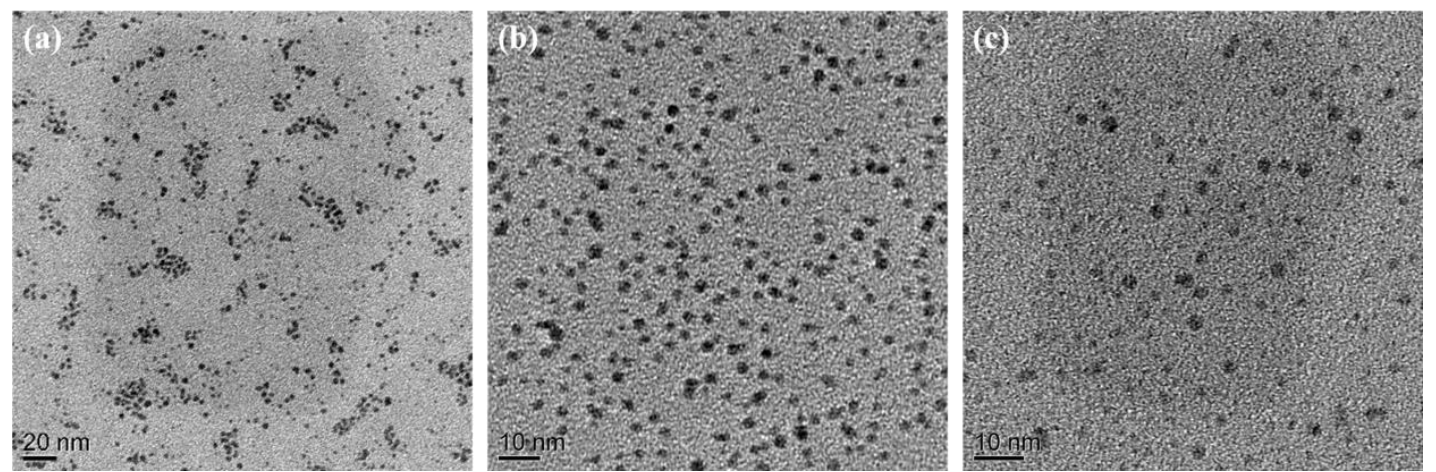

Figure S2. TEM images of (a) Pd@CSHPG, (b) Pd@DSHPG, and (c) Pd@OSHPG in nanofluids after 330 days. 


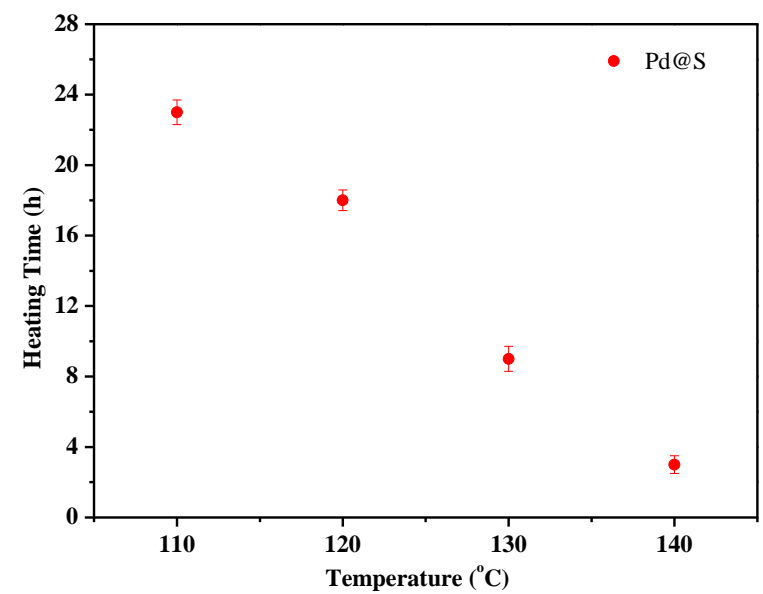

Figure S3. Thermal stability of nanofluids with $\mathrm{Pd} @ \mathrm{~S}$ at mass fraction of $0.1 \%$.

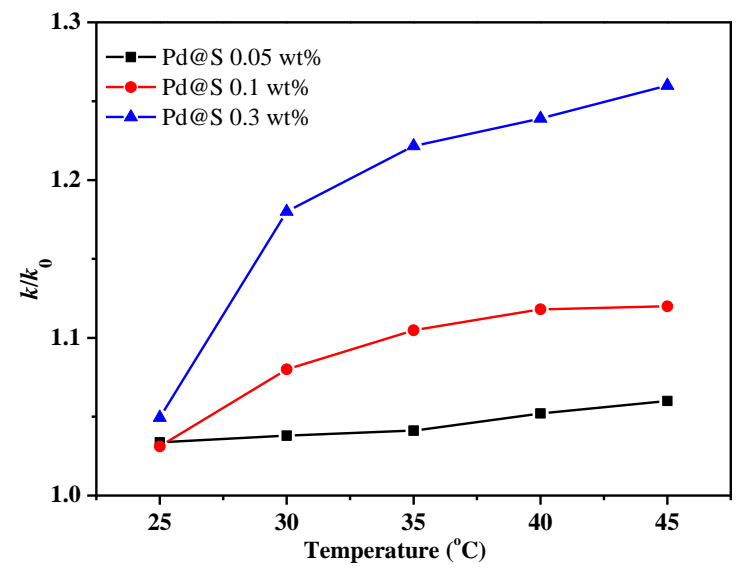

Figure S4. The ratio of thermal conductivity $\left(k / k_{0}\right)$ of nanofluids with Pd@S. 